\title{
Heavy absorption and soft X-ray emission lines in the XMM-Newton spectrum of the type 2 radio-loud quasar 3C 234
}

\author{
E. Piconcelli ${ }^{1}$, S. Bianchi ${ }^{2}$, G. Miniutti ${ }^{3}$, F. Fiore ${ }^{1}$, M. Guainazzi ${ }^{4}$, E. Jimenez-Bailon ${ }^{5}$, and G. Matt ${ }^{2}$ \\ ${ }^{1}$ Osservatorio Astronomico di Roma (INAF), via Frascati 33, 00040 Monteporzio Catone, Roma, Italy \\ e-mail: piconcelli@oa-roma.inaf.it \\ 2 Dipartimento di Fisica, Università degli Studi Roma 3, via della Vasca Navale 84, 00146 Roma, Italy \\ 3 Institute of Astronomy, Madingley Road, Cambridge CB3 OHA, UK \\ ${ }^{4}$ European Space Astronomy Center of ESA, Apartado 50727, 28080 Madrid, Spain \\ 5 Instituto de Astronomía, UNAM, Apartado 70264, 04510 Ciudad de México, Mexico
}

Received 27 September 2007 / Accepted 20 November 2007

\section{ABSTRACT}

\begin{abstract}
Aims. We report results on a $40 \mathrm{ks} X M M$-Newton observation of the type 2 quasar 3C 234. Optical spectropolarimetric data have demonstrated the presence of a hidden broad-line region in this powerful ( $M_{V} \leq-24.2$ after reddening and starlight correction) narrow-line FRII radio galaxy. Our analysis is aimed at investigating the X-ray spectral properties of this peculiar source that have remained poorly known so far.

Methods. We analyze the $0.5-10 \mathrm{keV}$ spectroscopic data collected by the EPIC cameras in 2006.

Results. The X-ray spectrum of this radio-loud quasar is typical of a local Compton-thin Seyfert 2 galaxy. It exhibits strong absorption $\left(N_{\mathrm{H}} \sim 3.5 \times 10^{23} \mathrm{~cm}^{-2}\right)$ and a narrow, neutral $\mathrm{Fe} \mathrm{K} \alpha$ emission line with an equivalent width of $\approx 140 \pm 40 \mathrm{eV}$. Our observation also reveals that the soft portion of the spectrum is characterized by strong emission lines with a very low level of scattered primary continuum. A possible explanation of these features in terms of thermal emission from a two-temperature, collisionally ionized plasma emission seems to be unlikely due to the high luminosity estimated for this component $\left(L_{0.5-2} \sim 6 \times 10^{42} \mathrm{erg} \mathrm{s}^{-1}\right)$. It is likely that most of the soft X-ray emission originates from a photoionized plasma as commonly observed in obscured, radio-quiet Seyfertlike AGNs.

Conclusions. This X-ray observation has definitively confirmed the presence of a hidden quasar in 3C 234. The line-rich spectrum and the steepness of the hard $\mathrm{X}$-ray continuum $(\Gamma \approx 1.7)$ found in this source weaken the hypothesis that the bulk of the $\mathrm{X}$-ray emission in radio-loud AGNs with high-excitation optical lines arises from jet non-thermal emission.
\end{abstract}

Key words. galaxies: active - galaxies: nuclei - quasars: individual: 3C 234 - X-rays: galaxies

\section{Introduction}

Type 2 quasars (QSO2s hereafter) are usually defined as those active galactic nuclei with the following properties: (i) a highly (i.e. $N_{\mathrm{H}} \geq 10^{22} \mathrm{~cm}^{-2}$ ) absorbed X-ray emission; (ii) an intrinsic, hard X-ray (i.e. $2-10 \mathrm{keV}$ ) luminosity $\geq 10^{44} \mathrm{erg} \mathrm{s}^{-1}$; and (iii) the lack of broad emission lines in their optical/UV spectra. The latter condition is often overcome since these objects are usually too weak, i.e. $R \gtrsim 25 \mathrm{mag}$, for optical spectroscopy. The QSO2 are therefore the luminous counterpart of Seyfert 2 galaxies. Even if their existence was postulated by the AGN unification model (Antonucci 1993; Urry \& Padovani 1995), QSO2 have represented for many years the long-sought missing AGN population (Halpern et al. 1999).

Since strongly biased by obscuration, optical and soft X-ray (0.5-2 keV) surveys fail to detect clearcut examples of such objects. However, over the past $\sim 10$ years, a very limited number of genuine QSO2s have been discovered (e.g. Brandt et al. 1997; Franceschini et al. 2000; Norman et al. 2002; Stern et al. 2002; Della Ceca et al. 2003; Iwasawa et al. 2005; Gandhi et al. 2006) thanks to X-ray observations above $2 \mathrm{keV}$. In particular, the recent deep and wide-area hard X-ray surveys performed with Chandra and XMM-Newton (e.g. Mainieri et al. 2002; Fiore et al. 2003; Brandt \& Hasinger 2005, Eckart et al. 2006;
Wang et al. 2007; Georgantopolous et al. 2007; Lacy et al. 2007) have efficiently detected several dozen QSO2 candidates. Unfortunately, most of them are sources with weak optical emission, showing a very high $\mathrm{X}$-ray-to-optical flux ratio $(\mathrm{X} / \mathrm{O}>10)$ and, therefore, with no reliable information about their redshift and classification (e.g. Maiolino et al. 2006). Furthermore, these hard X-ray surveys have established that the obscured AGN fraction decreases with increasing hard X-ray luminosity (Ueda et al. 2003; Steffen et al. 2003; La Franca et al. 2005; Akylas et al. 2006) and strongly increases with the hard X-ray flux only at $F_{2-10} \lesssim 10^{-14} \mathrm{erg} \mathrm{cm}^{-2} \mathrm{~s}^{-1}$ (Piconcelli et al. 2003). The combination of these findings explains the difficulties met so far in collecting a large sample of intrinsically-luminous, heavily obscured AGNs. According to the most up-to-date version of the synthesis model of the cosmic X-ray background (CXB), e.g. Gilli, Comastri \& Hasinger (2007), the population of obscured QSOs accounts for $\sim 15 \%$ of the CXB in the $2-10 \mathrm{keV}$ band.

Selecting sources with high [OIII] luminosity (i.e. $L_{\text {[OIII] }} \gtrsim 10^{42} \mathrm{erg} \mathrm{s}^{-1}$ ) (Derry et al. 2003; Vignali et al. 2006; Ptak et al. 2006) or with AGN luminosities in the mid-infrared and faint optical/near-infrared emission (MartinezSansigre et al. 2005; Houck et al. 2005) are the two alternative approaches that have been proven to be efficient in discovering large samples of QSO2s candidates. 
However, given the faintness in the $0.5-10 \mathrm{keV}$ band $\left(F_{0.5-10} \lesssim 10^{-14} \mathrm{erg} \mathrm{cm}^{-2} \mathrm{~s}^{-1}\right)$ of most of the QSOs candidates detected so far, only for a handful of these objects has it been possible to perform meaningful X-ray spectroscopy and, therefore, obtain tight constraints on the absorbing column density and, in general, on their X-ray properties, which have not been explored much so far (e.g. Akiyama et al. 2002; Severgnini et al. 2006; Piconcelli et al. 2007a).

In this paper, we present a $\sim 40 \mathrm{ks} X M M$-Newton observation of 3C 234, a radio galaxy with Fanaroff-Riley II edge-brightened morphology at $z=0.1848$ (Riley \& Pooley 1975). 3C 234 was initially classified as a broad-line radio galaxy owing to the presence of a weak broad $\mathrm{H} \alpha$ emission line in its optical spectrum (Grandi \& Osterbrock 1978). However, Antonucci (1982) and Antonucci (1984) found that both the optical/UV continuum and the broad $\mathrm{H} \alpha$ emission are highly polarized at a position angle perpendicular to the radio axis (with evidence that the broad line is more polarized than the continuum), while the narrow emission lines are very strong $\left(L_{[\mathrm{OIII}]} \sim 1.5 \times 10^{43} \mathrm{erg} \mathrm{s}^{-1}\right)$ and unpolarized. Furthermore, indications of strong extinction can be inferred by the absence of the blue-UV emission bump and the large broad-line Balmer decrement. On the basis of these findings, Antonucci \& Barvainis (1990) interpreted the polarization in terms of scattered light from the obscured continuum and broad-line region by a population of hot electrons and/or opaque dust clouds (e.g. Kishimoto et al. 2001, for more details) and proposed a narrow-line radio galaxy (NLRG) classification for this source. Tran et al. (1995, T95 hereafter) present the analysis of high signal-to-noise $(\mathrm{S} / \mathrm{N})$ Keck spectropolarimetric data for 3C 234 and confirm that the broad lines are caused by scattered light (Young et al. 1998; Cohen et al. 1999). Applying corrections for reddening and starlight contamination, T95 infer an absolute magnitude of $M_{V} \leq-24.2$, i.e. well within the quasar range. All these pieces of evidence indicate that 3C 234 harbors a buried luminous quasar in the nucleus i.e. this source is a type 2 quasar. Although affected by a low $\mathrm{S} / \mathrm{N}$, the ASCA spectrum of 3C 234 (Sambruna et al. 1999) provided further support to this classification suggesting the presence of strong absorption $\left(N_{\mathrm{H}} \sim\right.$ $\left.10^{23} \mathrm{~cm}^{-2}\right)$, an ultra-flat continuum $(\Gamma \sim 0.07)$, and a $2-10 \mathrm{keV}$ luminosity of $\sim 10^{44} \mathrm{erg} \mathrm{s}^{-1}$.

We adopt a $\Lambda$ CDM cosmology with $H_{0}=70 \mathrm{~km} \mathrm{~s}^{-1} \mathrm{Mpc}^{-1}$ and $\Omega_{\Lambda}=0.73$ (Spergel et al. 2007). For 3C 234 at $z=0.1848$, 1 arcsec corresponds to a physical scale of $3.1 \mathrm{kpc}$.

\section{XMM-Newton observation and data reduction}

The quasar 3C 234 was observed by XMM-Newton (Jansen et al. 2001, and reference therein) on April 24, 2006 for $\sim 40 \mathrm{ks}$. Data were reduced with SAS 6.5 using standard procedures. The X-ray events corresponding to patterns $0-12(0-4)$ for the $\operatorname{MOS}(P N)$ cameras were selected. The event lists were filtered to ignore periods of high background flaring according to the method presented in Piconcelli et al. (2004) based on the cumulative distribution function of background light curve count rates. After this data cleaning, we obtained a net exposure time of $34.5 \mathrm{ks}$ for the $P N$ and of $\sim 38 \mathrm{ks}$ for the two MOS cameras. The source spectra were extracted using a circular region of 25 and 32 arcsec radius for $P N$ and $M O S$, respectively, centered on the peak of the X-ray emission at $\alpha_{2000}=10^{\mathrm{m}} 01^{\mathrm{m}} 49.8^{\mathrm{s}}$ and $\delta_{2000}=+28^{\circ} 47^{\prime} 08^{\prime \prime}$. The $P N(M O S)$ background was estimated from a source-free region with a radius of 66(50) arcsec in the same chip. Response matrices and ancillary response files were generated using the RMFGEN and ARFGEN tools in the
SAS. Combined MOS spectrum and response matrix were created. Events outside the $0.5-10 \mathrm{keV}$ range were discarded in the $P N$ spectrum, while we ignored MOS data below the $0.8 \mathrm{keV}$ due to the presence of cross-calibration uncertainties between the MOS cameras (Kirsch 2006). Both $P N$ and MOS spectra were grouped to have a minimum of 20 counts per bin to apply the $\chi^{2}$ minimization technique, and fitted simultaneously.

During the observation the source flux remained steady, with no variation exceeding $2 \sigma$ from the average count-rate level in both soft- and hard-X-ray band and no significant spectral changes. Therefore our spectral analysis was performed on the spectrum integrated over the whole good exposure time.

\subsection{Spectral analysis}

All fits were performed using the XSPEC package (v11.3) and included absorption due to the line-of-sight Galactic column density of $N_{\mathrm{H}}=1.91 \times 10^{20} \mathrm{~cm}^{-2}$ (Dickey \& Lockman 1990). Hereafter, the quoted errors on the derived model parameters correspond to a $90 \%$ confidence level for one interesting parameter (i.e. $\Delta \chi^{2}=2.71$ ). Best-fit parameter values are given in the source frame, unless otherwise specified.

As a first step, we fitted the EPIC spectra at energies above $3 \mathrm{keV}$ using a simple power-law (PL) model in order to estimate the "hardness" of the X-ray continuum in 3C 234. This fit was very poor $\left(\chi_{v}^{2} \sim 3\right)$, with a ultra-flat photon index of $\Gamma \approx-0.4 \mathrm{de}-$ noting the complexity of the X-ray emission in this source and, in particular, the presence of strong absorption. We then fitted the 3-10 keV spectrum with a model consisting of a combination of a PL (representing the primary continuum) absorbed by neutral matter at the redshift of the source and a narrow Gaussian line to account for the $\mathrm{Fe} \mathrm{K} \alpha$ emission at $6.4 \mathrm{keV}$. This fit (hereafter indicated as baseline model) provides an excellent representation of the hard-band data, i.e. $\chi_{v}^{2}$ (d.o.f.) $=1.04(162)$, revealing an X-ray primary continuum with slope $\Gamma \approx 1.5$ affected by a large amount of intrinsic absorption $\left(N_{\mathrm{H}} \approx 3 \times 10^{23} \mathrm{~cm}^{-2}\right)$.

The Fe $\mathrm{K} \alpha$ emission line shows a rest-frame energy of $E_{\mathrm{K} \alpha}=$ $6.38_{-0.04}^{+0.03} \mathrm{keV}$ and an equivalent width $E W_{\mathrm{K} \alpha} \approx 140 \pm 40 \mathrm{eV}$. We obtained an upper limit on the line width of $\sigma_{\mathrm{K} \alpha}<120 \mathrm{eV}$.

The extrapolation of this spectral model to the soft X-ray band showed the presence of several narrow emission features along with a smooth excess below $\sim 3 \mathrm{keV}$ (e.g. Fig. 1). Such narrow features in the soft X-ray portion of the spectrum were initially modeled by the addition of a thermal plasma component (mekal in XSPEC) to the hard X-ray baseline model, under the hypothesis of an origin from starburst activity. This fit was completely unable to model the data at $E<3 \mathrm{keV}$ with an associated $\chi_{v}^{2} \sim 6$. We then added a second mekal component yielding a reasonable fit $\left(\chi_{v}^{2}(\right.$ d.o.f. $\left.)=1.16(255)\right)$. However, some large data/model residuals remained around $0.8-0.9 \mathrm{keV}$ (rest-frame). Furthermore, the temperature (and the $0.5-10 \mathrm{keV}$ luminosity of $\approx 2 \times 10^{43} \mathrm{erg} \mathrm{s}^{-1}$ as well) measured for one of the thermal components, i.e. $k T \sim 8 \mathrm{keV}$, seems unlikely for a starburst region (Franceschini et al. 2003). It can be an artifact of the fit related to the difficulty of fitting the soft excess in the $\sim 1.5-$ $3 \mathrm{keV}$ band. Accordingly, we included an additional unabsorbed PL component fixing its photon index to that of the absorbed PL, as expected in the case of scattered emission. The presence of the additional PL is required by the data at the $\gtrsim 99.8 \%$ confidence level. The temperatures of the two thermal components were $k T \approx 0.2$ and $k T \approx 0.6 \mathrm{keV}$. The application of this model (e.g. Table 1, model A) to the data produces a pretty acceptable fit to the EPIC data with an associated $\chi_{v}^{2}$ (d.o.f.) $=1.11(254)$ 


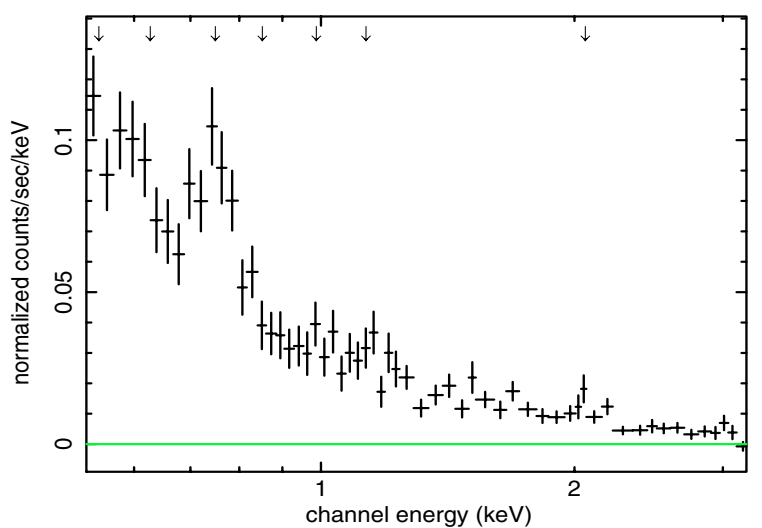

Fig. 1. The soft X-ray portion of the $P N$ spectrum of 3C 234 when the hard X-ray baseline model (i.e. absorbed power-law continuum + narrow $\mathrm{Fe} \mathrm{K} \alpha$ line) is applied to the $0.5-10 \mathrm{keV}$ band. A large soft X-ray excess dominated by several emission line-like features is seen. The arrows indicate the observer-frame energy of the significantly detected emission lines as listed in Table 2.

but leaves evident line-like positive residuals below $1 \mathrm{keV}$ (see Fig. 1).

We then performed a phenomenological fit adding to the baseline model seven narrow Gaussian lines to account for the emission features observed in the $\sim 0.5-2 \mathrm{keV}$ range. Each Gaussian line is required at a significance level of $P_{F}>99.9 \%$ (except for the line at $2.44 \mathrm{keV}$ with $P_{F}=98.5 \%$ ). However, due to the potential line blending with adjacent emission lines, such a detection significance should be considered with care, and in some cases, it may represent only an upper limit. The photon index of the unabsorbed PL $\left(\Gamma_{\text {soft }}\right)$ was left free to vary since blends of unresolved emission lines appear as a pseudocontinuum when observed at the spectral resolution of EPIC. This PL component also accounts for a mixture of emissions caused by, i.e., the electron-scattered fraction of the primary continuum, thermal and/or photoionized plasma, the relativisic jet, and Compton reflection off ionized matter (see Miniutti et al. 2007; Evans et al. 2006a; Turner et al. 1997), as observed in most of X-ray obscured AGNs. We yielded a very good description of the spectrum of 3C 234 (e.g. model B in Table 1), with an associated $\chi_{v}^{2}$ (d.o.f.) $=1.04(246)$. The confidence contours for $\Gamma$ versus $N_{\mathrm{H}}$ are plotted in Fig. 3. The ratio between the normalizations of the unabsorbed and absorbed PL is $f_{\mathrm{s}} \approx 0.03$. In Table 2 the best-fit parameters are listed together with the likely identification for each of the soft X-ray lines.

Finally, the observed flux in the $2-10 \mathrm{keV}$ band is $F_{2-10} \sim 1.3 \times 10^{-12} \mathrm{erg} \mathrm{cm}^{-2} \mathrm{~s}^{-1}$, which is consistent with the value measured with ASCA in 1994 (Sambruna et al. 1999).

\section{Discussion}

\subsection{The hard X-ray spectrum and the Fe Ko emission line}

The EPIC observation presented here is the first good-quality $\mathrm{X}$-ray spectrum of 3C 234 and, in general, it represents one of the best X-ray observation collected for a genuine QSO2 so far. Our analysis reveals the presence of a complex spectrum, largely dominated by strong obscuration. The X-ray continuum shows a slope of $\Gamma \sim 1.7$ and is absorbed by Compton-thin $\left(N_{\mathrm{H}} \sim 3 \times\right.$ $10^{23} \mathrm{~cm}^{-2}$ ) neutral gas. Since the intrinsic $2-10 \mathrm{keV}$ luminosity is $L_{2-10} \sim 3 \times 10^{44} \mathrm{erg} \mathrm{s}^{-1}, 3 \mathrm{C} 234$ satisfies all the conditions to be considered a genuine type 2 quasar.
The value of the photon index of the continuum is consistent with the average value found for other lobe-dominated ${ }^{1}$ FRII quasars, i.e. $\langle\Gamma\rangle \sim 1.6-1.7$ (Belsole et al. 2006; Piconcelli et al. 2005; Grandi et al. 2006). Harder photon indices of $\Gamma \sim 1.4-$ 1.5 are indeed usually observed in the core-dominated, morealigned radio-loud AGNs, the so-called Blazars, whose featureless X-ray spectrum is dominated by relavistically-beamed inverse-Compton jet emission (e.g. Fossati et al. 1998; Donato et al. 2005). The XMM-Newton measurement implies that the jet contribution to the broad-band X-ray emission must be marginal as suggested by the detection of many soft X-ray emission lines, as well as a prominent $\mathrm{Fe} \mathrm{K} \alpha$ line at $6.4 \mathrm{keV}$ in its spectrum. It is worth noting that this is the first time that such an emission line has been detected at a statistically significant level in $3 \mathrm{C} \mathrm{234.} \mathrm{The} \mathrm{best-fit} \mathrm{rest-frame} \mathrm{energy} \mathrm{of} \mathrm{the} \mathrm{Fe} \mathrm{K} \alpha$ line is $E_{\mathrm{K} \alpha}=6.38_{-0.04}^{+0.03} \mathrm{keV}$, corresponding to low ionization states, i.e. FeI-XVI (Kallman et al. 2004). The $E W \approx 140 \mathrm{eV}$ of this line matches the value predicted by theoretical calculations well (e.g. Awaki et al. 1991; Leahy \& Creighton 1993; Ghisellini et al. 1994) for an $\mathrm{Fe} \mathrm{K} \alpha$ line produced by transmission through an absorbing screen with a column of $N_{\mathrm{H}} \sim 3-4 \times 10^{23} \mathrm{~cm}^{-2}$, as what is measured along the line of sight to the nucleus of 3C 234 by the XMM-Newton observation. This suggests that such a Compton-thin absorber is responsible for both obscuration and fluorescent narrow $\mathrm{Fe} \mathrm{K} \alpha$ emission. However, the value of the EW is also consistent with reflection off distant Comptonthick matter (Guainazzi et al. 2005). The cold reflector could then be located at the far inner side of the molecular torus invoked in the AGN unified models (Antonucci 1993; see also Elitzur \& Shlosman 2006) and seen along an unobscured line of sight.

This scenario implies the simultaneous presence of two circumnuclear obscuring gases, one Compton-thick (the reflector) and one Compton-thin (the absorber), as recently discovered in some Seyfert 2 galaxies showing large X-ray spectral variations (Matt et al. 2003; Risaliti et al. 2005; Piconcelli et al. 2007b). If this is the case, the Compton-thin matter should cover only a small fraction of the sky (as seen by the X-ray primary source) in order not to produce too much iron $\mathrm{K} \alpha$ emission (Matt 2002). Furthermore the $\mathrm{Fe} \mathrm{K} \alpha$ line should be accompanied by a Compton-reflection continuum emission peaking at $\sim 30 \mathrm{keV}$ (Matt, et al. 1991; Krolik et al. 1994). Unfortunately, the limited EPIC bandpass does not allow the strength of this spectral component in 3C 234 to be meaningfully constrained, and, in turn, test the validity of this hypothesis. Future spectroscopy in the 10-60 keV range, say with Simbol- $X$ (Ferrando et al. 2006), will therefore be crucial for distinguishing a transmission from a reflection origin for the $\mathrm{Fe} \mathrm{K} \alpha$ line in this QSO2.

Young et al. (1998) inferred a dust extinction toward 3C 234 of $A_{\mathrm{V}}=60 \mathrm{mag}$, which corresponds to an absorbing column density of $\approx 1.1 \times 10^{23} \mathrm{~cm}^{-2}$, if the standard Galactic gasto-dust ratio is assumed (i.e. $N_{\mathrm{H}} / A_{\mathrm{V}}=1.79 \times 10^{21} \mathrm{~cm}^{-2} \mathrm{mag}^{-1}$, Predehl \& Schmitt 1995), which is a factor of $\sim 3.4$ lower than observed. This mismatch has been observed in many X-ray sources as reported by Maiolino et al. (2001), who proposed the existence of dust grains larger than in the diffuse Galactic medium that do not redden the optical emission efficiently. Alternatively, Weingartner \& Murray (2002) suggest that X-ray

\footnotetext{
1 Lobe-dominated radio-loud sources are those with a rest frame ratio (the so-called core dominance $R_{\mathrm{c}}$ ) of radio core to lobe flux density of $\log R_{\mathrm{c}}<0$ (Orr \& Brown 1982). According to Sambruna et al. (1999) $3 \mathrm{C} 234$ has a $\log R_{\mathrm{c}}=-1.34$.
} 
Table 1. Best-fit spectral parameters of the EPIC spectrum. See Sect. 2.1 for details.

\begin{tabular}{cccccccccc}
\hline \hline $\begin{array}{c}\text { Model } \\
\text { (1) }\end{array}$ & $\begin{array}{c}\Gamma \\
(2)\end{array}$ & $\begin{array}{c}N_{\mathrm{H}} \\
(3)\end{array}$ & $\begin{array}{c}f_{s} \\
(4)\end{array}$ & $\begin{array}{c}\Gamma_{\text {Soft }} \\
(5)\end{array}$ & $\begin{array}{c}k T \\
(6)\end{array}$ & $\begin{array}{c}\text { Flux } \\
(7)\end{array}$ & $\begin{array}{c}\text { Luminosity } \\
(8)\end{array}$ & $\begin{array}{c}E W_{\mathrm{K} \alpha} \\
(9)\end{array}$ & $\begin{array}{c}\chi^{2} \text { (d.o.f.) } \\
(10)\end{array}$ \\
\hline (A) & $1.53_{-0.04}^{+0.03}$ & $3.12_{-0.17}^{+0.17}$ & 0.05 & $\equiv \Gamma$ & $0.63_{-0.07}^{+0.06}$ & $0.83 / 13.27$ & $0.06^{*} / 2.61$ & $139_{-40}^{+42}$ & $282(254)$ \\
& & & & $0.17_{-0.01}^{+0.01}$ & & & & & \\
(B) & $1.71_{-0.04}^{+0.05}$ & $3.69_{-0.18}^{+0.19}$ & 0.03 & $1.09_{-0.09}^{+0.13}$ & - & $0.85 / 13.18$ & $1.62 / 2.97$ & $135_{-41}^{+43}$ & $255(246)$ \\
\hline
\end{tabular}

The columns give the following information: (1) model (A: baseline model + two mekal components + unabsorbed PL, B: baseline model + soft X-ray Gaussian lines + unabsorbed PL); (2) photon index of the absorbed continuum power law; (3) the column density of the absorber $\left(10^{23} \mathrm{~cm}^{-2}\right)$; (4) ratio between the normalization of the unabsorbed versus absorbed PL component at $1 \mathrm{keV}$; (5) photon index of the unabsorbed soft X-ray power law; (6) the temperature $(\mathrm{keV})$ of the thermal plasma component (mekal); (7) the 0.5-2/2-10 keV observed flux $\left(10^{-13} \mathrm{erg} \mathrm{cm}^{-2} \mathrm{~s}^{-1}\right)$; (8) the $0.5-2 / 2-10 \mathrm{keV}$ luminosity $\left(10^{44} \mathrm{erg} \mathrm{s}^{-1}\right)$; (9) $E W$ of the Fe $\mathrm{K} \alpha$ line (eV); (10) $\chi^{2}$ and number of degrees of freedom. ${ }^{*}$ Luminosity of the thermal emission component in the $0.5-2.0 \mathrm{keV}$ band.
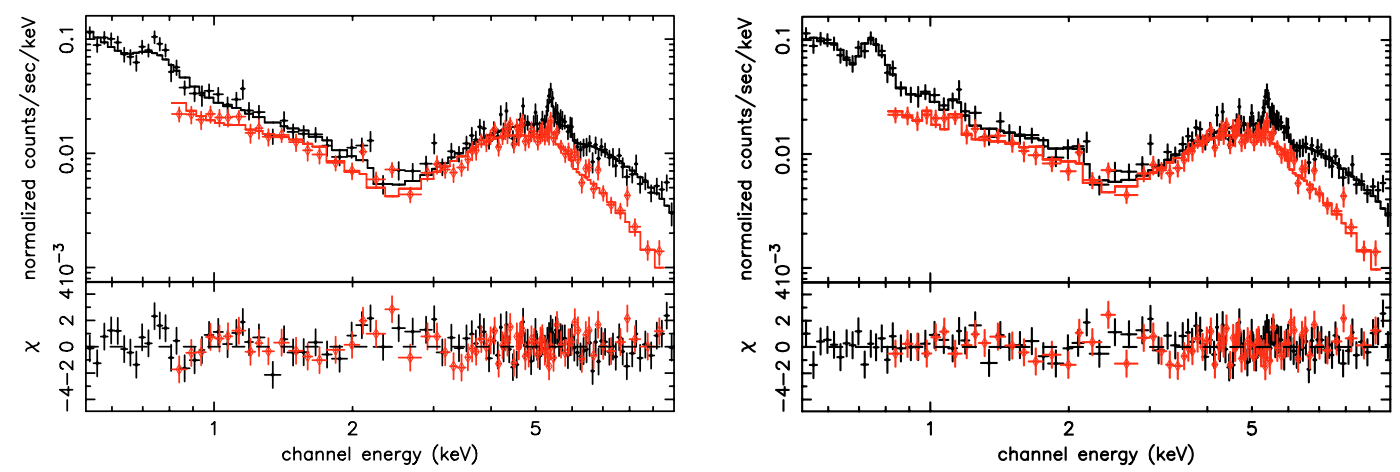

Fig. 2. a) Left: XMM-Newton PN (top) and MOS (bottom) spectra of 3C 234 when the model A is applied. The lower panel shows the deviations of the observed data from the model in unit of standard deviation. b) Right: the same as in the left figure but for model B. See Table 1 for the best-fit parameters of each model.

absorption and optical extinction occur in distinct regions, with the X-ray absorber located inside the dust sublimation radius.

\subsubsection{The [Mg VIII] to $2-10 \mathrm{keV}$ luminosity ratio}

Imanishi (2006) report detecting a strong high-excitation emission line at $3.028 \mu \mathrm{m}$ due to [Mg VIII], a hallmark of the presence of a powerful AGN in this source. We derive an [Mg VIII] to 2-10 keV luminosity ratio $\mathrm{Mg}^{7+} / \mathrm{X}=0.004$ assuming an [Mg VIII] luminosity $L_{\mathrm{Mg}^{7+}}=1.2 \times 10^{42} \mathrm{erg} \mathrm{s}^{-1}$. Noteworthy is that such a ratio is similar to the one inferred for the archetypical Seyfert 2 galaxy Circinus, i.e. $\mathrm{Mg}^{7+} / \mathrm{X}=0.005$ using an $L_{\mathrm{Mg}^{7+}}=4.75 \times 10^{39} \mathrm{erg} \mathrm{s}^{-1}$ (Sturm et al. 2002) and $L_{2-10}=10^{42} \mathrm{erg} \mathrm{s}^{-1}$ (Matt et al. 1999), despite the $L_{\mathrm{Mg}^{7+}}$ of the QSO2 being approximatively a factor of 250 larger than in Circinus. A value of $\mathrm{Mg}^{7+} / \mathrm{X}=0.004$ is also found for the heavily Compton-thick source NGC $1068\left(L_{\mathrm{Mg}^{7+}}=3.1 \times 10^{40} \mathrm{erg} \mathrm{s}^{-1}\right.$; Marco \& Brooks 2003) if we adopt a $L_{2-10}=7.7 \times 10^{42} \mathrm{erg} \mathrm{s}^{-1}$, i.e. the value determined by Levenson et al. (2006) from the Fe $\mathrm{K} \alpha$ line luminosity (see also Panessa et al. 2006). The possibility of using $L_{\mathrm{Mg}^{7+}}$ as a proxy for the intrinsic X-ray luminosity of an obscured active nucleus deserves further investigation; therefore, a larger sample of AGN is needed to confirm this relationship on sounder statistical grounds. Unfortunately, an accurate measurement of the intensity [Mg VIII] emission line at $3.028 \mu \mathrm{m}$ is available to date in the literature only for a handful of sources.

\subsection{The Soft $X$-ray emission}

The emission lines detected for the first time at $\$ 2 \mathrm{keV}$ in the XMM-Newton spectrum of 3C 234 (see Table 2) rule out the

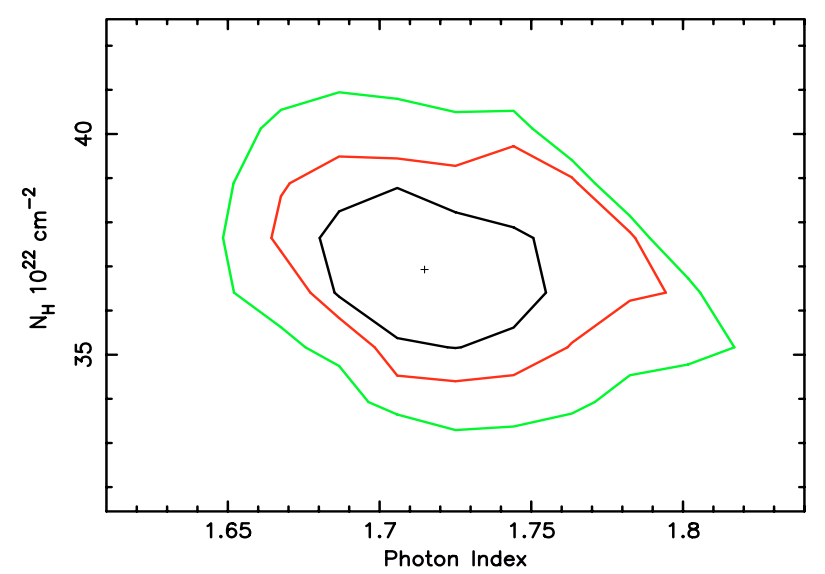

Fig. 3. Confidence contour plot showing the intrinsic rest-frame column density against the hard X-ray continuum photon index. The contours are at $68 \%, 90 \%$, and $99 \%$ confidence levels for two interesting parameters.

hypothesis that the bulk of the soft X-ray emission arises in this radiogalaxy from jet-related non-thermal emission, as suggested by Evans et al. (2006a) and Belsole et al. (2006) by the analysis of X-ray observations of narrow-line radio-loud AGNs. It is worth noting that a simple fit with an unabsorbed PL in the 0.5$2 \mathrm{keV}$ band yields a very steep photon index $(\Gamma \sim 2.6)$, which agrees with slopes of the soft X-ray PLs in absorbed FR II radio galaxies as reported by Belsole et al. (2006).

Furthermore, as mentioned in Sect. 2.1, the 0.5-2 keV luminosity of $\approx 5.8 \times 10^{42} \mathrm{erg} \mathrm{s}^{-1}$ of the collisionally-heated plasma component (mekal in XSPEC) inferred by model A is too large to be associated with starburst regions, which typically show 
Table 2. Best-fit spectral parameters for the emission lines detected in the soft X-ray band.

\begin{tabular}{cccc}
\hline \hline $\begin{array}{c}\text { Energy } \\
(1)\end{array}$ & $\begin{array}{c}\text { Intensity } \\
(2)\end{array}$ & $\begin{array}{c}P_{F} \\
(3)\end{array}$ & $\begin{array}{c}\text { Identification } \\
(4)\end{array}$ \\
\hline $0.65_{-0.01}^{+0.01}$ & $17.2_{-2.7}^{+4.9}$ & $>99.9$ & OVIII Ly $\alpha$ \\
$0.74_{-0.02}^{+0.01}$ & $11.1_{-1.9}^{+2.1}$ & $>99.9$ & OVII RRC, Fe XVII 3s-2p \\
$0.89_{-0.01}^{+0.01}$ & $12.3_{-1.9}^{+1.2}$ & $>99.9$ & OVIII RRC, NeIX He $\alpha$, \\
& & & Fe XVIII 3d-2p \\
$1.02_{-0.02}^{+0.01}$ & $1.4_{-0.9}^{+0.6}$ & $>99.9$ & NeX Ly $\alpha$, Fe XXI 3d-2p \\
$1.17^{\dagger}$ & $1.8_{-0.5}^{+0.6}$ & $>99.9$ & FeXXIV L, NeX Ly $\beta$, \\
& & & NeIX RRC \\
$1.34^{\dagger}$ & $1.4_{-0.5}^{+0.5}$ & $>99.9$ & MgXI He $\alpha$ \\
$2.44^{\dagger}$ & $0.4_{-0.1}^{+0.3}$ & 98.5 & SXV He $\alpha$ \\
\hline
\end{tabular}

The columns give the following information: (1) energy of the line $(\mathrm{keV})$; (2) intensity of the line $\left(10^{-6}\right.$ photons $\left.\mathrm{cm}^{-2} \mathrm{~s}^{-1}\right)$; (3) significance of the fit improvement after including the Gaussian line in the fitting model (based on $F$-test, see Sect. 2.1 for further details on this significance value); (4) likely identification (e.g. Brinkman et al. 2002; Guainazzi \& Bianchi 2007). ${ }^{\dagger}$ Indicates that the parameter has been fixed to the best-fit value deduced by the fit.

$10^{39} \lesssim L_{0.5-2} \lesssim$ a few times $10^{41}$ erg s$^{-1}$ (e.g. Ptak et al. 1999; Franceschini et al. 2003). Using the relationship between farinfrared (FIR) and soft X-ray luminosity reported by Ranalli et al. (2003) for a large sample of star-forming galaxies, we estimate a starburst luminosity of $L_{0.5-2}^{\mathrm{SB}} \lesssim 3.3 \times 10^{41} \mathrm{erg} \mathrm{s}^{-1}$, i.e. a contribution $\$ 4 \%$ to the total soft $X$-ray observed luminosity in $3 \mathrm{C} 234\left(L_{0.5-2}^{\mathrm{Obs}}=8.2 \times 10^{42} \mathrm{erg} \mathrm{s}^{-1}\right)$, assuming an FIR luminosity of $L_{\mathrm{FIR}} \lesssim 1.54 \times 10^{45} \mathrm{erg} \mathrm{s}^{-1}$. According to Kennicutt (1998) the FIR luminosity of 3C 234 implies a star-formation rate of $S F R<70 M_{\odot} \mathrm{yr}^{-1}$, while a $L_{0.5-2}=5.8 \times 10^{42} \mathrm{erg} \mathrm{s}^{-1}$ translates into an $S F R \sim 1300 M_{\odot} \mathrm{yr}^{-1}$ (Ranalli et al. 2003), i.e. a very high value that is only observed in the most massive nuclear starbursts triggered by strong tidal interactions and mergers of galaxies. This finding agrees with the results of Imanishi (2006) that in the 3-4 $\mu$ ( $L$-band) spectrum of 3C 234 found $3.3 \mu \mathrm{m}$ emission PAH features more than an order of magnitude weaker than those of typical starburst galaxies.

As the soft X-ray emission is not affected by absorption, the location of the emitting gas in this QSO2 must be far away from the obscuring gas intercepting the primary continuum. The soft X-ray emission lines found by XMM-Newton can be associated (bearing in mind the EPIC resolution and the error bars of the energy values listed in Table 2) with hydrogen- and helium-like lines of the most abundant light metals, from oxygen to sulfur. This result is very similar to those reported from the XMM-Newton and Chandra observations of obscured radioquiet Seyfert-like AGNs (e.g. Guainazzi \& Bianchi 2007, and references therein), in which the soft X-ray emission is dominated by a wealth of emission lines and spatially coincident with the extended [OIII] emission (Iwasawa et al. 2003; Bianchi et al. 2006). High-resolution X-ray grating spectroscopy of this soft $\mathrm{X}$-ray emission provides unambiguous evidence that most of it is produced in a photoionized outflowing gas (e.g. Kinkhabwala et al. 2002; Guainazzi \& Bianchi 2007). On the other hand, the exact physical mechanism generating the emission-line spectra is still debated. Both AGN-photoionization and "local" photoionization due to gas heated by high-speed shocks driven by nuclear outflows (i.e. the radio jet) in the interstellar medium (Fu \& Stockton 2006; Evans et al. 2006b) are viable mechanisms.
This scenario could likely hold also in 3C 234 even if this source is a radio-loud QSO2. In particular, the detection of two prominent lines at 0.74 and $0.88 \mathrm{keV}$ bolsters this hypothesis since they may be associated with narrow OVII and OVIII radiative recombination continuum (RRC), respectively, which are hallmarks of recombination in a low-temperature photoionized plasma (Liedahl 1999). However, better quality high-resolution spectral data are necessary before a firm conclusion can be drawn on the exact origin of these emission lines. Due to the weakness of 3C 234 in the soft X-ray band $\left(F_{0.5-2} \approx 8 \times 10^{-14} \mathrm{erg} \mathrm{cm}^{-2} \mathrm{~s}^{-1}\right)$ no meaningful RGS data were collected during the present XMM-Newton observation. This does not allow estimation of the possible contribution arising from collisionally-ionized plasma.

The discovery of a rich emission-line soft X-ray spectrum in 3C 234 is noteworthy since this is the first time it has been found in a type 2 and, even more remarkably, a radio-loud QSO. In fact, Sambruna et al. (2007) and Grandi et al. (2007) have only very recently reported the first clearcut example of a radio-loud AGN with a soft X-ray spectrum dominated by emission lines on the basis of the XMM-Newton observation of the heavily absorbed FRII radio galaxy 3C 445 (e.g. also Young et al. 2002). These findings suggest that inner circumnuclear regions in radio-loud (in particular those with high excitation optical emission lines, which usually show an FRII radio morphology, e.g. Grandi \& Palumbo 2007; Ballantyne 2007) and radio-quiet AGNs could have similar geometrical and physical properties.

Finally, according to model B the unabsorbed PL has a photon index of $\Gamma_{\text {Soft }}=1.09_{-0.09}^{+0.13}$. The $0.5-2 \mathrm{keV}$ luminosity of this spectral component is $L_{0.5-2} \approx 4 \times 10^{42} \mathrm{erg} \mathrm{s}^{-1}$, which represents about $2-3 \%$ of the intrinsic luminosity of 3C 234 in the soft $\mathrm{X}$-ray band $\left(\approx 1.6 \times 10^{44} \mathrm{erg} \mathrm{s}^{-1}\right)$. This emission could be due to a blend of scattered nuclear emission arising from the lineemitting warm photoionized plasma (and/or the electron mirror located interior to the NLR proposed by T95 on the basis of spectropolarimetric data) and unresolved X-ray emitting knots of the kpc-scale jet (which usually show flat $\mathrm{X}$-ray slope, $\Gamma \approx 1.0-1.3$; e.g. Sambruna et al. 2004), plus a possible, marginal (i.e. $<8 \%$, assuming a starburst luminosity of $L_{0.5-2}^{\mathrm{SB}}<3.3 \times 10^{41} \mathrm{erg} \mathrm{s}^{-1}$ estimated from the FIR luminosity of 3C 234; e.g. Ranalli et al. 2003) contribution from X-ray binaries.

\section{Summary}

In this paper, we present analysis of the XMM-Newton observation of 3C 234 collected in 2006. These data provide the most detailed description to date of the X-ray spectral properties of this FRII radio galaxy, which has been long known for hosting a hidden quasar at its center (Antonucci \& Barvainis 1990).

The quality of the data allows light to be shed on many controversial issues about the characteristics of the X-ray emission in 3C 234, i.e. the exact shape of the continuum, the iron $\mathrm{K}$ fluorescent emission and the nature of the soft X-ray spectral component, left unsettled by the low S/N 1994 ASCA observation. We measure an $L_{2-10} \approx 3 \times 10^{44} \mathrm{erg} \mathrm{s}^{-1}$, whereby 3C 234 falls well within the quasar X-ray luminosity range (Fabian 2003). At face value, the $0.5-10 \mathrm{keV}$ spectrum of this radio-loud QSO2 is typical of a nearby Compton-thin Seyfert 2 galaxy. It is dominated by heavy obscuration $\left(N_{\mathrm{H}} \sim 3.7 \times 10^{23}\right.$ $\mathrm{cm}^{-2}$ ), with a strong, narrow $\mathrm{Fe} \mathrm{K} \alpha$ emission line at $6.4 \mathrm{keV}$ and many emission lines from highly ionized metals in the soft X-ray band. Such a line-rich spectrum and the steepness of the 
hard X-ray continuum, $\Gamma \approx 1.7$, reveal that the non-thermal emission arising from the relativistic jet provides a marginal contribution to the $\mathrm{X}$-ray emission. The strength of the $\mathrm{Fe} \mathrm{K} \alpha$ line is fairly consistent with what is expected in transmission from the observed obscuring screen with $N_{\mathrm{H}} \sim 4 \times 10^{23} \mathrm{~cm}^{-2}$. However, a line origin from reflection occurring at the AGN-illuminated, far inner surface of the Compton-thick torus cannot be rule out. This in turn would imply the presence of two absorbing regions coexisting in this QSO2.

The detection of lines at 0.74 and $0.87 \mathrm{keV}$ (i.e. the energy of the narrow OVII and OVIII RRC, respectively) strongly indicates that most of the soft X-ray emission originates from a photoionized plasma. The presence of this warm gas is particularly important since it suggests that the circumnuclear environment in radio-loud and radio-quiet AGNs share the same geometrical and physical properties. This remarkable finding leads support to recent XMM-Newton results (e.g. Grandi et al. 2007; Sambruna et al. 2007), which reported a similar scenario for $3 \mathrm{C} 445$, another X-ray luminous, obscured FRII radio galaxy.

Acknowledgements. We thank the anonymous referee for a number of helpful comments that improved the paper. The authors kindly thank Roberto Maiolino (OAR) for very useful discussions. EP is supported by an INAF Postdoctoral Fellowship. Based on observations obtained with XMM-Newton, an ESA science mission with instruments and contributions directly funded by ESA Member States and NASA. This research made use of the NASA/IPAC Extragalactic Database (NED), which is operated by the Jet Propulsion Laboratory, California Institute of Technology, under contract with the National Aeronautics and Space Administration. EP acknowledges financial contribution from contract ASI-INAF I/023/05/0.

\section{References}

Akiyama, M., Ueda, \& Y., Ohta, K. 2002, ApJ, 567, 42

Akylas, A., Georgantopoulos, I., Georgakakis, A., Kitsionas, S., \&

Hatziminaoglou, E. 2006, A\&A, 459, 693

Antonucci, R. 1982, Nature, 299, 605

Antonucci, R. 1984, ApJ, 278, 499

Antonucci, R. 1993, ARA\&A, 31, 473

Antonucci, R., \& Barvainis, R. 1990, ApJ, 363, L17

Avni, Y. 1976, ApJ, 210, 642

Awaki, H., Koyama, K., Inoue, H., \& Halpern, J. P. 1991, PASJ, 43, 195

Ballantyne, D. R. 2007, Modern Phys. Lett. A, in press,

[astro-ph/0707.3142]

Belsole, E., Worrall, D. M., \& Hardcastle, M. J. 2006, MNRAS, 366, 339

Bianchi, S., Guainazzi, M., \& Chiaberge, M. 2006, A\&A, 448, 499

Brandt, W. N., \& Hasinger, G. 2005, ARA\&A, 43, 827

Brandt, W. N., Fabian, A. C., Takahashi, K., et al. 1997, MNRAS, 290, 617

Brinkman, A. C., Kaastra, J. S., van der Meer, R. L. J., et al. 2002, A\&A, 396, 761

Cohen, M. H., Ogle, P. M., Tran, H. D., Goodrich, R. W., \& Miller, J. S. 1999, AJ, 118, 1963

Della Ceca, R., Braito, V., Beckmann, V., et al. 2003, A\&A, 406, 555

Derry, P. M., O'Brien, P. T., Reeves, J. N., et al. 2003, MNRAS, 342, 53

Dickey, J. M., \& Lockman, F. J. 1990, ARA\&A. 28, 215

Donato, D., Sambruna, R. M., \& Gliozzi, M. 2005, A\&A, 433, 1163

Eckart, M. E., Stern, D., Helfand, D. J., et al. 2006, ApJS, 165, 19

Elitzur, M., \& Shlosman, I. 2006, ApJ, 648, L101

Evans, D. A., Worrall, D. M., Hardcastle, M. J., Kraft, R. P., \& Birkinshaw, M. 2006a, ApJ, 642, 96

Evans, D. A., Lee, J. C., Kamenetska, M., et al. 2006b, ApJ, 653, 1121

Fabian, A. C. 2003, Carnegie Observatories Astrophysics Ser., vol. I, ed. L. Ho (Cambridge University Press) [arXiv: astro-ph/0304122]

Ferrando, P., Goldwurm, A., Laurent, P., et al. 2006, Proc. SPIE, 6266, 62660F

Fiore, F., Brusa, M., Cocchia, F., et al. 2003, A\&A, 409, 79

Franceschini, A., Bassani, L., Cappi, M., et al. 2000, A\&A, 353, 910

Franceschini, A., Braito, V., Persic, M., et al. 2003, MNRAS, 343, 1181

Fossati, G., Maraschi, L., Celotti, A., Comastri, A., \& Ghisellini, G. 1998, MNRAS, 299, 433

Fu, H., \& Stockton, A. 2006, ApJ, 650, 80

Gandhi, P., Fabian, A. C., \& Crawford, C. S. 2006, MNRAS, 369, 1566

Georgantopoulos, I., Georgakakis, A., \& Akylas, A. 2007, A\&A, 466, 823

Gilli, R., Comastri, A., \& Hasinger, G. 2007, A\&A, 463, 79

Ghisellini, G., Haardt, F., \& Matt, G. 1994, MNRAS, 267, 743

Grandi, P., \& Palumbo, G. G. C. 2007, ApJ, 659, 235
Grandi, P., Malaguti, G., \& Fiocchi, M. 2006, ApJ, 642, 113

Grandi, P., Guainazzi, M., Cappi, M., \& Ponti, G. 2007, MNRAS, 381, L21

Grandi, S. A., \& Osterbrock, D. E. 1978, ApJ, 220, 783

Guainazzi, M., \& Bianchi, S. 2007, MNRAS, 374, 1290

Guainazzi, M., Matt, G., \& Perola, G. C. 2005, A\&A, 444, 119

Halpern, J. P., Turner, T. J., \& George, I. M. 1999, MNRAS, 307, L47

Houck, J. R., Soifer, B. T., Weedman, D., et al. 2005, ApJ, 622, L105

Imanishi, M. 2006, AJ, 131, 2406

Iwasawa, K., Wilson, A. S., Fabian, A. C., \& Young, A. J. 2003, MNRAS, 345, 369

Iwasawa, K., Crawford, C. S., Fabian, A. C., \& Wilman, R. J. 2005, MNRAS, 362, L20

Jansen, F., Lumb, D., Altieri, B., et al. 2001, A\&A, 365, L1

Kallman, T. R., Palmeri, P., Bautista, M. A., Mendoza, C., \& Krolik, J. H. 2004, ApJS, 155, 675

Kennicutt, R. C. 1998, ARA\&A, 36, 189

Kinkhabwala, A., Sako, M., Behar, E., et al. 2002, ApJ, 575, 732

Kirsch, M. 2006, EPIC status of calibration and data analysis, XMM-SOC-CALTN-0018, Issue 2.5

Kishimoto, M., Antonucci, R., Cimatti, A., et al. 2001, ApJ, 547, 667

Krolik, J. H., Madau, P., \& Zycki, P. T. 1994, ApJ, 420, L57

La Franca, F., Fiore, F., Comastri, A., et al. 2005, ApJ, 635, 864

Lacy, M., Petric, A. O., Sajina, A., et al. 2007, AJ, 133, 186

Leahy, D. A., \& Creighton, J. 1993, MNRAS, 263, 314

Levenson, N. A., Heckman, T. M., Krolik, J. H., Weaver, K. A., \& Zycki, P. T. 2006, ApJ, 648, 111

Liedahl, D. A. 1999, in X-Ray Spectrocopy in Astrophysics, ed. J. van Paradijs,

\& J. A. M. Bleeker (Berlin: Springer), 189

Maiolino, R., Marconi, A., Salvati, M., et al. 2001, A\&A, 365, 28

Maiolino, R., Mignoli, M., Pozzetti, L., et al. 2006, A\&A, 445, 457

Mainieri, V., Bergeron, J., Hasinger, G., et al. 2002, A\&A, 393, 425

Marco, O., \& Brooks, K. J. 2003, A\&A, 398, 101

Martinez-Sansigre, A., Rawlings, S., Lacy, M., et al. 2005, Nature, 436, 666

Matt, G. 2002, MNRAS, 337, 147

Matt, G., Perola, G. C., \& Piro, L. 1991, A\&A, 247, 25

Matt, G., Guainazzi, M., Maiolino, R., et al. 1999, A\&A, 341, L39

Matt, G., Guainazzi, M., \& Maiolino, R. 2003, MNRAS, 342, 422

Miniutti, G., Ponti, G., Dadina, M., Cappi, M., \& Malaguti, G. 2007, MNRAS, 375,227

Norman, C., Hasinger, G., Giacconi, R., et al. 2002, ApJ, 571, 218

Orr, M. J. L., \& Browne, I. W. A. 1982, MNRAS, 200, 1067

Panessa, F., Bassani, L., Cappi, M., et al. 2006, A\&A, 455, 173

Piconcelli, E., Cappi, M., Bassani, L., Di Cocco, G., \& Dadina, M. 2003, A\&A, 412, 689

Piconcelli, E., Jimenez-Bailon, E., Guainazzi, M., et al. 2004, MNRAS, 351, 161

Piconcelli, E., Guainazzi, M., Cappi, M., Jimenez-Bailon, E., \& Schartel, N. 2005, A\&A, 432, 835

Piconcelli, E., Fiore, F., Nicastro, F., et al. 2007a, A\&A, 473, 85

Piconcelli, E., Bianchi, S., Guainazzi, M., Fiore, F., \& Chiaberge, M. 2007b, A\&A,466, 855

Predehl, P., \& Schmitt, J. H. M. M. 1995, A\&A, 293, 889

Ptak, A., Serlemitsos, P., Yaqoob, T., \& Mushotzky, R. 1999, ApJS, 120, 179

Ptak, A., Zakamska, N. L., Strauss, M. A., et al. 2006, ApJ, 637, 147

Ranalli, P., Comastri, A., \& Setti, G. 2003, A\&A, 399, 39

Riley, J. M., \& Pooley, G. G. 1975, MmRAS, 80, 105

Risaliti, G., Elvis, M., Fabbiano, G., Baldi, A., \& Zezas, A. 2005 ApJ, 623, L93

Tran, H. D., Cohen, M.H., \& Goodrich, R. W. 1995, AJ, 110, 2597

Turner, T. J., George, I. M., Nandra, K., \& Mushotzky, R. F. 1997, ApJS, 113, 23

Sambruna, R. M., Eracleous, M., \& Mushotzky, R. F. 1999, ApJ, 526, 60

Sambruna, R. M., Gambill, J. K., Maraschi, L., et al. 2004, ApJ, 608, 698

Sambruna, R. M., Reeves, J. N., \& Braito, V. 2007, ApJ, 665, 1030

Severgnini, P., Caccianiga, A., Braito, V., et al. 2006, A\&A, 451, 859

Spergel, D.N., Bean, R., Doré, O., et al. 2007, ApJS, 170, 377

Steffen, A. T., Barger, A. J., Cowie, L. L., Mushotzky, R. F., \& Yang, Y. 2003, ApJ, 596, L23

Stern, D., Moran, E. C., Coil, A. L., et al. 2002, 568, 71

Sturm, E., Lutz, D., Verma, A., et al. 2002, A\&A, 393, 821

Tran, H. D., Cohen, M. H., \& Goodrich, R. W. 1995, AJ, 110, 2597 (T95)

Ueda, Y., Akiyama, M., Ohta, K., \& Miyaji, T. 2003, ApJ, 598, 886

Urry, C. M., \& Padovani, P. 1995, PASP, 107, 803

Vignali, C., Alexander, D. M., \& Comastri, A. 2006, MNRAS, 373, 321

Wang, J. X., Jiang, P., Zheng, Z. Y., et al. 2007, ApJ, 657, 95

Weingartner, J. C., \& Murray, N. 2002, ApJ, 580, 88

Young, S., Hough, J. H., Axon, D. J., Fabian, A. C., \& Ward, M. J. 1998, MNRAS, 294, 478

Young, A. J., Wilson, A. S., Terashima, Y., Arnaud, K. A., \& Smith, D. A. 2002, ApJ, 564, 176 